\title{
LES MÉLANOMES MALINS NASOSINUSIENS
}

\author{
SINONASAL MELANOMA
}

\author{
I. HARIGA, S. ZRIBI, CH. M'BAREK, M. KEMERJI, O. BEN GAMRA, I. CHEDLI*, M. BEN AYED*, \\ A. EL KHEDIM \\ SERVICE ORL ET DE CCF \\ * SERVICE D'ANATOMOPATHOLOGIE \\ HÔPITAL HABIB THAMEUR DE TUNIS
}

\begin{abstract}
RESUME
Le mélanome malin nasosinusien est une tumeur rare prenant naissance au niveau des mélanocytes de la muqueuse respiratoire. Les auteurs rapportent deux observations de mélanomes malins nasosinusiens suivis et traités au service d'ORL et de CCF de l'hôpital Habib Thameur entre 1999 et 2005. Il s'agit d'un homme et d'une femme âgés respectivement de 62 et 68 ans. La symptomatologie clinique est dominée par l'obstruction nasale et l'épistaxis. Le diagnostic est histologique après biopsie de la tumeur. Le traitement chirurgical est suivi d'une radiothérapie externe dans les deux cas. L'évolution est marquée par une récidive tumorale dans un cas.
\end{abstract}

\section{SUMMARY}

Primary mucosal melanoma of the nasal cavity and paranasal sinuses is an uncommun clinical entity occurring on the level of the melanocytes respiratory mucous membrane. The authors report two observations of malignant melanoma of sinonasal mucosa treated between 1999 and 2005. It acts of an old man and a woman respectively 62 and 68 years old, both presented with nasal obstruction and epistaxis. The diagnosis was histological after biopsy of the tumour. The surgical treatment was followed of an external radiotherapy in both cases. The evolution was marked by a local recurrence in one case.

\section{INTRODUCTION}

La localisation naso-sinusienne des mélanomes malins est rare. Elle ne dépasse pas $2 \%$ de l'ensemble des mélanomes $(1,2,3)$. Néanmoins elle est la première localisation muqueuse de la tête et du cou. Ces mélanomes dérivent des mélanocytes présents dans l'épithélium respiratoire des fosses nasales et des sinus. Leur siège préférentiel est le cornet inférieur, rarement le cornet moyen. L'âge de survenue est nettement plus élevé que celui des les mélanomes cutanés. Le diagnostic est histologique, renforcé par l'immunohistochimie devant certaines variétés achromiques. L'imagerie moderne est incontournable pour un bilan précis locorégional. Le traitement est essentiellement chirurgical. Le pronostic est sombre, amélioré par une prise en charge précoce est multidisciplinaire.

\section{MATERIEL ET METHODES}

Nous rapportons deux observations de mélanomes malins nasosinusiens diagnostiqués et pris en charge au service d'ORL et de chirurgie cervicofaciale de l'hôpital Habib Thameur entre 1999 et 2005.

\section{Observation 1 :}

Madame F. T. âgée de 68 ans, a consulté pour une obstruction nasale unilatérale gauche évoluant depuis 6 mois associée à une épistaxis homolatérale et des céphalées.

L'endoscopie nasale a objectivé une formation tissulaire polyploïde noirâtre ferme occupant la totalité de la fosse nasale gauche. Le reste de l'examen était normal, en particulier pas d'adénopathies cervicales et pas de lésions cutanées.

La tomodensitométrie du massif facial a révélé un comblement tissulaire de la fosse nasale gauche s'étendant aux choanes avec un comblement partiel du sinus maxillaire gauche.

L'examen histologique après une biopsie de la tumeur était en faveur d'un carcinome épidermoïde.

Le bilan d'extension était négatif, classant la tumeur en un stade 1. Le bilan biologique était normal.

Le traitement a consisté en une exérèse chirurgicale large par la voie de Rouge Dunker.

L'examen anatomopathologique définitif a conclu à un mélanome malin avec des cellules égrainées, de multiples mitoses ainsi que des grains de pigment intracellulaires. L'immunohistochimie est positive à l'anticorps S 100 et au HMB 45.

Une radiothérapie externe à la dose de 50 gray a été délivrée 4 mois après le traitement chirurgical.

La patiente est perdue de vue après 3 ans de suivi.

\section{Observation 2 :}

Monsieur A.M. âgé de 62 ans, a consulté pour une obstruction nasale unilatérale gauche et épistaxis évoluant depuis 5 mois. 
L'endoscopie nasale a objectivé une tumeur bourgeonnante noirâtre comblant la fosse nasale gauche, les choanes et le cavum qui saigne au contact, sans adénopathies palpables. L'examen cutané était normal.

La tomodensitométrie a montré une formation tissulaire comblant la fosse nasale gauche jusqu'aux choanes et s'étendant vers les sinus maxillaire et ethmoïdal gauches. Une lyse osseuse de la cloison intersinusonasale a était notée (Fig.1,2)
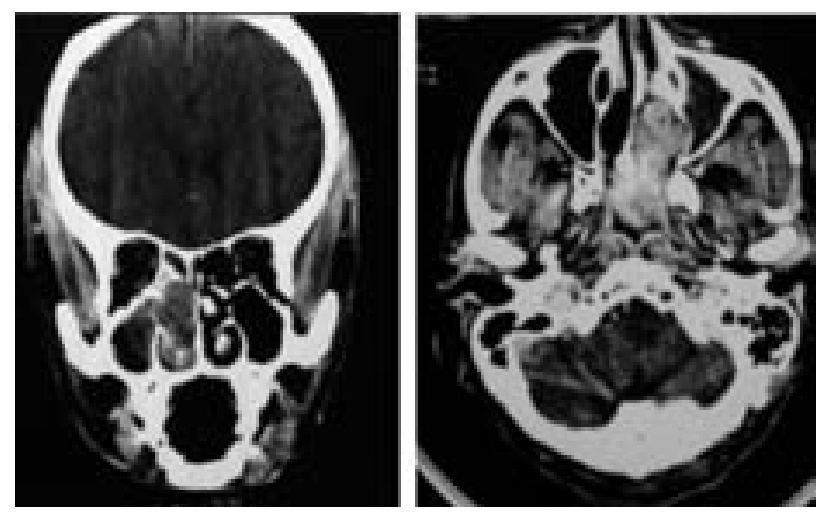

Fig. 1 et 2 : Formation tumorale de la fosse nasale gauche, arrivant aux choanes et s'étendant au sinus maxillaire et l'ethmoïde $G$ avec lyse de la cloison intersinusonasale

L'examen anatomopathologique de la biopsie a conclu à un mélanome malin grâce à la présence d'une immunoréactivité intense des cellules tumorales vis à vis des anticorps anti HMB 45 et anti PS 100. Le bilan d'extension à distance est négatif en particulier la scintigraphie osseuse est normale. La tumeur est classée stade 1.

Le traitement chirurgical a consisté en une ethmoïdo maxillectomie gauche avec une sphenoidotomie homolatérale par voie transfaciale (Fig.3). Un évidement ganglionnaire sus omohyoidien gauche a était réalisé dans le même temps opératoire.

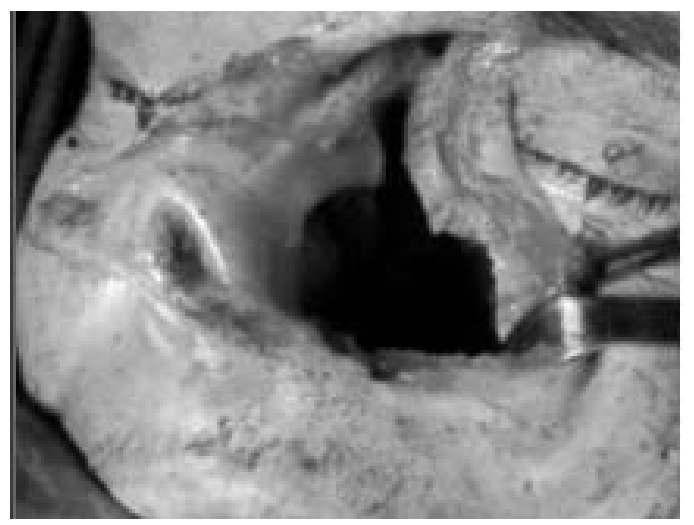

Fig. 3 : Vue per-opératoire après exérèse

L'examen anatomopathologique a confirmé le diagnostic de mélanome malin sans métastase ganglionnaire.

Un traitement adjuvant par radiothérapie externe a été réali- sé à la dose de 54 Gy. Le contrôle tomodensitométrique était satisfaisant (Fig.4,5).

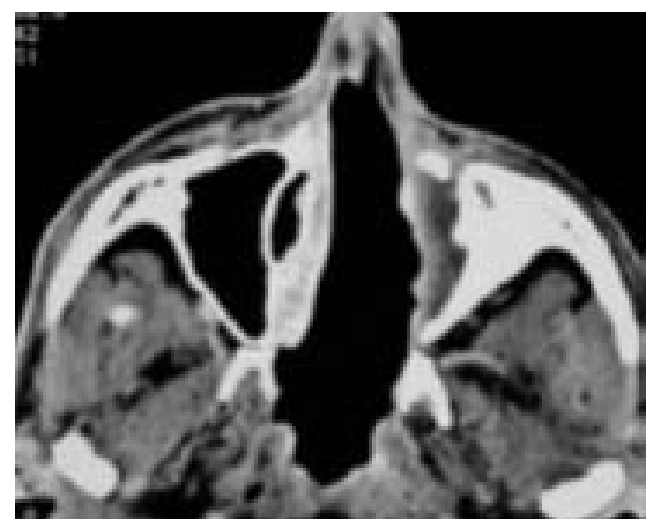

Fig. 4 : TDM Post opératoire

Six mois après la fin de la radiothérapie le patient présente une adénopathie jugulo carotidienne haute gauche. Une adénectomie a été réalisée. L'examen histologique a conclu à un aspect réactionnel. Deux ans plus tard, une récidive locale a necessité la reprise de la voie paralatéronasale. L'imagerie postopératoire était rassurante. L'évolution a été émaillée par une poursuite évolutive avec exophtalmie gauche et une métastase vertébrale lombaire. Le patient a reçu une irradiation flash au niveau du rachis de D11 à L3 de 8 Gy puis a été perdu de vue.

\section{DISCUSSION}

Le mélanome malin est une prolifération néoplasique faite de cellules d'origine neuroectodermique de type mélanocyte avec ou sans pigment mélanique (4).

Les mélanomes nasosinusiens représentent 1 à $2 \%$ de tous les mélanomes et $3,6 \%$ à $12 \%$ des tumeurs malignes nasosinusiennes $(1,4,5,6)$.

C'est une pathologie du sujet âgé avec un âge moyen qui varie de 50 à 70 ans dans la littérature $(4,5,7,8)$. La répartition selon le sexe est variable selon les séries $(1,4,8)$. Aucune prédominance raciale n'a été constatée.

Au niveau des fosses nasales, la tumeur intéresse fréquemment le septum, la paroi latérale des fosses nasales et les cornets. Mais souvent le point de départ de la tumeur est difficile à préciser devant l'extension très rapide de la tumeur. Le sinus maxillaire est le premier sinus atteint $(7,9)$.

L'étiopathogénie est mal élucidée mais certains facteurs ont été incriminés tel que le tabac et le formaldehide. L'exposition au soleil ne joue aucun rôle dans la survenue de ces mélanomes muqueux.

La symptomatologie clinique est dominée par l'obstruction nasale et l'épistaxis souvent unilatérale.

L'endoscopie nasale objective une masse bourgeonnante molasse hémorragique gris noirâtre ou ardoisée qui comble la fosse nasale. Les formes achromiques représentent $20 \%$ des mélanomes $(1,3)$.

L'examen clinique doit vérifier l'absence de mélanome cutané. 
La fréquence des métastases ganglionnaires au moment du diagnostic varie entre 0 et $23 \%$. Celle des métastases à distance est de 0 à $14 \%(8,9)$.

Les tests biologiques spécifiques, recherche de la protéine $\mathrm{H} 700$, dosage de l'hémopexine sérique, autrefois signalés (10), ne sont plus de pratique courante.

Cavalier a démontré en 1980 la prédominance du type HLA A9 chez les sujets atteints de mélanomes malins (in 10). Cette hypothèse n'a pas été retrouvée dans la littérature récente.

Le diagnostic est histologique. II repose sur la mise en évidence de la mélanine en intracellulaire. Dans les formes achromiques le diagnostic se fait par le microscope électronique, par la mise en évidence des prémélanosomes, ou par l'immunohistochimie objectivant une réaction positive avec l'antivimentine, l'anti S 100 et l'anti HMB 45(2,4).

L'extension tumorale est essentiellement locale et la majorité des patients sont classés stade au moment du diagnostic. L'imagerie par résonance magnétique précise les extensions de la tumeur à l'orbite, à la fosse infratemporale et au sinus caverneux. Les rapports osseux sont étudiés à la tomodensitométrie $(8,9)$.

Le traitement est avant tout chirurgical. II repose sur l'exérèse chirurgicale large justifiée par la multifocalité de la tumeur et l'amélioration du pronostic par le contrôle local de la tumeur. La voie d'abord doit être large permettant une résection complète avec une marge de sécurité de $2 \mathrm{~cm}(3,4,8,9)$. La chirurgie endonasale sous guidage endoscopique peut être proposée pour les tumeurs localisées à la partie antéroinférieure des fosses nasales et ne dépassant pas $1 \mathrm{~cm}(4)$. La radiothérapie externe fractionnée est utilisée seule ou après la chirurgie avec des résultats controversés.

La chimiothérapie est utilisée comme traitement adjuvant dans les formes évoluées avec des résultats décevants $(9,11,12)$.
D'autres moyens ont été utilisés : l'immunothérapie par le $B C G$ et le traitement chimiohormonal par andro œstrogènes sans résultats concluants $(4,8)$.

Les récidives locales sont très fréquentes, observées dans 40 à $80 \%$ des cas et précédant généralement les métastases ganglionnaires et les métastases à distance $(4,9)$.

La surveillance est capitale basée sur l'endoscopie nasale et l'imagerie. Actuellement certains auteurs font recours à la scintigraphie par iode 123 BZA et le dosage de l'interleukine 6.

Le pronostic est redoutable avec une survie à 5 ans qui varie de 10 à $47 \%(2,4,9,11,12)$. II est statistiquement corrélé au stade de la tumeur au moment du diagnostic, à l'épaisseur de la tumeur (supérieur à $0,5 \mathrm{~mm}$ ) et à l'invasion vasculaire (13). Une étude récente a montré une corrélation entre le taux de la protéine oncocytaire la c-myc et la prolifération tumorale (14). Les tumeurs nasales sont de meilleur pronostic que les tumeurs sinusiennes.

\section{CONCLUSION}

Le mélanome malin nasosinusien est une pathologie rare, dont le tableau clinique est non spécifique. Le diagnostic est histopathologique et immunohistochimique. Le traitement chirurgical doit être radical. L'amélioration du pronostic passe par un diagnostic précoce et un contrôle local de la tume.

\section{REFERENCES}

1- LE CLECH G. Mélanomes malins cervico-faciaux. Encycl Med Chir oto-rhinolaryngologie (Paris), 20- 950- E- 10, 2000.

2- MANOLIDIS S., DONALD PJ. Malignant mucosal melanoma of the head and neck. Cancer. 1997; 80: 1373- 86.

3- RICHARD J.M, Guerrier Y., Les tumeurs du massif facial supérieur. Actualités de carcinologie cervico-faciale. Masson 1991.

4- KHAROUBI S. Mélanome malin des fosses nasales : considérations cliniques et thérapeutiques à propos de trois cas. Cancer/Radiothérapie $2005 ; 9$ : 99-103. 5- DEMATOS P. TYLER DS. SEIGLER H F. Malignant melanoma of the mucous membranes: a review of 119 cas. Ann Serg Oncol. 1998: 5: 733- 42.

$6-$ Noel T. Ciu, MARTIN A. Melanoma of oronasal mucosa. Arch Otolaryngol Head Neck Surg. 1996; 122: 985- 988.

7- LORREE TR. MULLINS AP. SPELLMAN J. NORTH JH Jr. Head and neck mucosal melanoma: a 32 years review. Ear Nose Throat J 1999; 78: 78-37. 8- SNEHAL G. PATEL, MANJU L. PRASAD, MARGARITA ESCRIG and al. Primary mucosal malignant melanoma of the Head and neck. Head and Neck; $2002: 247-256$.
9- CASTILLO L., POISSONNET G., HADDAD A. et al. Mélanomes malins nasosinusiens : bilan et stratégie thérapeutique. Cahier d'ORL 1998 ;XXXIII n8 :42632.

10-A. EL KHEDIM, A. BEN HASSINE, A. LADGHAM and al .mélanomes malins primitifs de la sphère ORL. Jr. Tun. ORL - Vol. 1 - No 3 - Juin 1985

11- PERALTA EA, YARINGTON TC, GLENN MG. Malignant melanoma of the head and neck. Laryncoscope 1998;108:220-3

12- LEE SP, SHIMIZUKT, TRAN LM, JUILLARD G et al. mucosal melanoma of the Head and neck: effect of treatment on survival. Larynncoscope 1998;108:155-61.

13- Patel SG, PRASAD ML, ESCRIG M and al. Long Term follow-up of c-myc, p53 and proliferation measurements in malignant melanoma

14- ROSS D.A., LAING J.H.E. SANDERS R. AND WILSON G.D. Long term follw -up of c-myc,p53 and proliferation measurements in malignant melanoma. Euro. J. of Surgical oncology; 2006:80-84. 